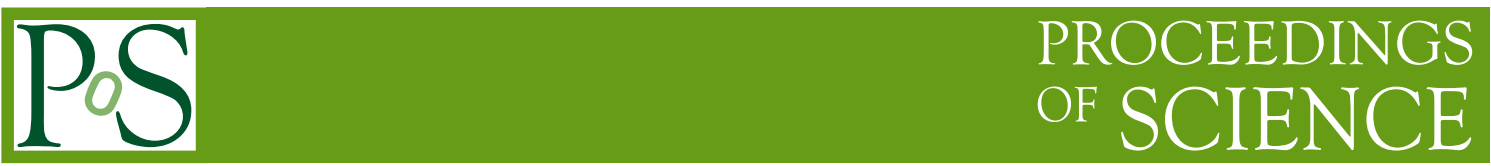

\title{
Pion/Kaon Decay in Very Special Relativity
}

\section{Pankaj Jain*}

Department of Physics, Indian Institute of Technology,Kanpur

E-mail: pkjain@iitk.ac.in

\section{Alekha Chandra Nayak}

Department of Physics, Indian Institute of Technology, Kanpur

E-mail: acnayakeitk.ac.in

We consider the charged pion decay process within the framework of Very Special Relativity (VSR) using an effective Lagrangian approach. We construct an effective Lagrangian for this process which is invariant under VSR but violates Lorentz symmetry. We find that in the rest frame of pion, the final state muon distribution is not isotropic due to VSR contributions. In the laboratory frame, the decay products show azimuthal angle dependence relative to the pion beam momentum. Furthermore the differential cross section picks up a periodic time dependence with a period of 1 sidereal day. Although such effects are expected in Lorentz violating (LV) theories, this is the first illustration that they also arise within the framework of VSR. This is interesting since VSR effects are most likely to observed at low energies in contrast with all other models of LV which are expected to be significant only at very high energies. We argue that VSR leads to such effects in many high energy processes and not just the pion decay.

9th International Workshop on the CKM Unitarity Triangle

28 November - 3 December 2016

Tata Institute for Fundamental Research (TIFR), Mumbai, India

\footnotetext{
* Speaker.
} 


\section{Introduction}

Very Special Relativity (VSR) is based on the idea that the frame independence of the speed of light does not necessarily require Lorentz invariance. It only demands that a subgroup, such as $\mathrm{T}(2), \mathrm{E}(2), \operatorname{HOM}(2)$ and $\operatorname{SIM}(2)$ remains preserved [1]. Here we shall be interested primarily in the subgroups $\operatorname{HOM}(2)$ and $\operatorname{SIM}(2)$. Let $\mathbf{J}$ and $\mathbf{K}$ represent generators of rotations and boosts respectively. The generators of $\operatorname{HOM}(2)$ are $T_{1}=K_{x}+J_{y}, T_{2}=K_{y}-J_{x}$ and $K_{z}$ and those of $\operatorname{SIM}(2)$ are $T_{1}, T_{2}, J_{z}$ and $K_{z}$. This possibility is allowed as long as the discrete symmetries P, T, CP (or CT) are broken. It turns out that the dispersion relations of particles as well as several consequences of Special relativity, such as time dilation and velocity addition formula, remain unchanged in this framework [1]. Phenomenological consequences of VSR have been studied in [2, 3, 4, 5, 6, 7, 8, 9, $10,11,12,13,14]$.

The possibility of violation of Lorentz invariance also arises within the framework of many quantum gravity models. For example, it is argued that at very high energy scales of order Planck mass $\left(M_{P l}\right)$, space-time coordinates may not commute with one another [15, 16]. Such Lorentz violations (LV) are expected to contribute dominantly at very high energies. However it has been argued that these may induce rather large effects through loop corrections which to be cancelled by fine tuning of parameters [17]. It is interesting that supersymmetry naturally cancels these large corrections $[18,19]$ and predicts LV of order $M_{S U S Y}^{2} / M_{P l}^{2}$, at energy scales currently accessible in laboratory. Here $M_{S U S Y}$ is the scale of SUSY breaking. At energy scales exceeding $M_{S U S Y}$ we expect $\mathrm{LV}$ of order $E^{2} / M_{P l}^{2}$. In contrast, the LV effects due to VSR may give dominant contribution at low energies, as we describe below.

\section{Effective Lagrangian for pion decay in the VSR framework}

The Lorentz violating (LV) terms which respect VSR can be constructed by defining a null vector

$$
n^{\mu}=(1,0,0,1)
$$

This vector is invariant under the $T_{1}$ and $T_{2}$ transformations but picks up an overall factor under $K_{z}$. Hence it is not invariant under $\operatorname{HOM}(2)$ and $\operatorname{SIM}(2)$. Using this vector we can construct a nonlocal LV term which respects these symmetry groups. Consider a Standard Model (SM) fermion multiplet $\psi$ which may be left or right handed. We may add the following LV term to the SM action,

$$
\mathscr{L}_{V S R}=\frac{i \tilde{m}^{2}}{2} \bar{\psi} \frac{n \cdot \gamma}{n \cdot D} \psi
$$

for each of the SM fermion multiplets. Here $D$ is the gauge covariant derivative. These terms lead to LV, VSR invariant masses for all the SM fermions and furthermore lead to LV, VSR invariant interactions of fermions with all the gauge bosons $[9,13,20]$. This introduces additional mass parameters for each left and right SM fermion multiplets. For the case of leptons this parameter is limited by the neutrino mass. Furthermore there exist very stringent constraints on the spin dependent mass term for the case of electron. In order to satisfy this constraint it has been argued that we should impose $\mathrm{C}$ invariance on such mass terms which requires equal mass parameters for left and right handed multiplets [3]. Since weak interactions break C invariance, loop corrections 
will generate such a mass difference for electron even if we set it equal to zero at the tree level. Such corrections will arise as long as such LV fermion mass terms are included in the action. Furthermore if these mass parameters are sufficiently large, we may need to fine tune the left-right mass difference in order to evade experimental constraints. In our analysis we shall ignore this fine tuning problem and aim to impose limits on VSR mass parameters for each fermion multiplet directly from experimental data. We point out that we do not do far have a fundamental theory based on VSR. Furthermore loop corrections in the presence of such nonlocal terms lead to infrared divergences, which are not easily handled. A reasonable prescription for handling such divergences has been suggested in [20] which may allow a detailed phenomenological study of these models.

Here we are interested in the LV, VSR invariant effects for simple processes such as charged pion decay. Similar effects are expected to arise in many other high energy processes. The pion decay process proceeds through a $W$ exchange, as shown in Fig. 1. The LV contributions arise due the VSR corrections to the quark masses and in the W-quark vertex. We ignore the LV contributions in the lepton sector. Calculation of this amplitude requires the pion wave function, which is not known. An estimate of this loop can be made by using the linear sigma model for strong interaction [20]. This leads to an effective coupling between the pion and leptons which can be expressed in terms of a VSR invariant effective Lagrangian, given by,

$$
L=V_{u d} \frac{G_{f}}{\sqrt{2}} \mathfrak{f}_{\pi} \partial_{\mu} \Pi \bar{\psi} \gamma^{\mu}\left(1-\gamma^{5}\right) \psi+\bar{g}\left(\frac{n_{\mu}}{n . \partial} \Pi\right) \bar{\psi} \gamma^{\mu}\left(1-\gamma^{5}\right) \psi+\text { h.c. }
$$

Here $\Pi$ is the pion field and $\bar{g}$ is the Lorentz violating coefficient. In Eq. 2.3, the first term produces the SM decay rate and the second term gives VSR contribution with effective coupling parameter $\bar{g}$.

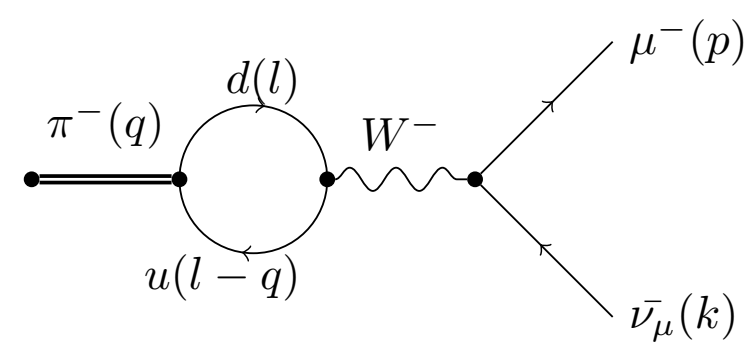

Figure 1: The Feynman diagram for the decay of charged pion in a lepton pair.

\section{Laboratory Signatures of Lorentz Violation}

We first consider the decay of pion in its rest frame. We can always go to the rest frame by making SIM(2) transformation. The decay rate can be directly computed by using the effective Lagrangian 2.3. We find that the differential decay rate leads to a angular dependence of the final state muon distribution due to the LV contributions [20]. We also impose an upper limit on the parameter $\bar{g}$ by assuming that VSR contributes less than the error in measurement in life time of pion. The resulting upper limit is found to be $\bar{g}=2.1 \times 10^{-12} \mathrm{GeV}$. 
We next consider the decay process in the laboratory frame. We find several interesting effects which can be tested experimentally. In particular, the final state muon distribution shows an azimuthal angle dependence. Here the azimuthal angle is defined by choosing the beam axis as the $z$-axis. The polar angle distribution of muons also gets modified due to the VSR effects. Furthermore the peak position in the azimuthal distribution shows a periodic dependence on time with a period of 1 sidereal day. The polar angle distribution also shows a correlated time dependence. This time dependence of the distribution can be used to effectively test the VSR contributions [20] in elementary particle experiments.

To conclude, VSR invariant Lorentz violating extension of the Standard Model leads to several interesting predictions which can be tested in high energy particle colliders. Using an effective field theory framework we have shown that the final state particles in the case of pion decay process show anisotropic, time dependent angular distributions. These can be studied experimentally in order to impose a direct limit on the VSR contributions to the up and down quark masses.

\section{References}

[1] A. G. Cohen and S. L. Glashow, Phys. Rev. Lett. 97, 021601 (2006).

[2] A. G. Cohen and S. L. Glashow (2006), hep- ph/0605036.

[3] A. Dunn and T. Mehen, arXiv:hep-ph/0610202.

[4] J. Fan, W. D. Goldberger, and W. Skiba, Phys. Lett. B649, 186 (2007).

[5] A. G. Cohen and D. Z. Freedman, JHEP 07, 039 (2007).

[6] G. W. Gibbons, J. Gomis, and C. N. Pope, Phys. Rev. D76, 081701 (2007).

[7] A. E. Bernardini and R. da Rocha, Europhys. Lett. 81, 40010 (2008).

[8] M. M. Sheikh-Jabbari and A. Tureanu, Phys. Rev. Lett. 101, 261601 (2008).

[9] S. Cheon, C. Lee, and S. J. Lee, Phys. Lett. B679, 73 (2009).

[10] D. V. Ahluwalia and S. P. Horvath, JHEP 11, 078 (2010).

[11] J. Vohánka, Phys. Rev. D 85, 105009 (2012).

[12] J. Alfaro and V. O. Rivelles, Phys.Rev. D88, 085023 (2013).

[13] J. Alfaro and P. González and R. Ávila, Phys. Rev. D91, 105007 (2015)

[14] C.-Y. Lee, Phys. Rev. D93, 045011 (2016).

[15] S. Doplicher and K. Fredenhagen and J. E. Roberts, Phys. Lett. B 331, 39 (1994).

[16] D. V. Ahluwalia, Phys. Lett. B 339, 301 (1994).

[17] J. Collins, A. Perez, D. Sudarsky, L. Urrutia, and H. Vucetich, Phys. Rev. Lett. 93, 191301 (2004).

[18] S. Groot Nibbelink and M. Pospelov, Phys. Rev. Lett. 94, 081601 (2005).

[19] P. Jain and J. P. Ralston, Phys. Lett. B621, 213 (2005).

[20] A. C. Nayak and P. Jain, arXiv:1610.01826. 